\title{
BMJ Open Risk factors for bone metastasis in patients with primary lung cancer: study protocol for a systematic review
}

\author{
Yu-Jie Niu, Yin-Tian Wen, Wei-Wei Shen, Lin Deng, Li-Li Liu, He-Long Zhang
}

To cite: Niu Y-J, Wen Y-T, Shen W-W, et al. Risk factors for bone metastasis in patients with primary lung cancer: study protocol for a systematic review. BMJ Open 2014:4:e005202.

doi:10.1136/bmjopen-2014005202

- Prepublication history and additional material is available. To view please visit the journal (http://dx.doi.org/ 10.1136/bmjopen-2014005202).

Received 6 March 2014 Revised 20 June 2014 Accepted 7 July 2014
Department of Oncology, Tangdu Hospital, The Fourth Military Medical University, Xi'an, China

Correspondence to He-Long Zhang; cnxazh@163.com

\section{ABSTRACT}

Introduction: Bone metastasis (BM) in patients with primary lung cancer poses a serious health problem. Numerous risk factors have been hypothesised to predict BM in these patients, but research studies are of mutable quality, and may not be of value in clinical evaluation.

Methods and analysis: We will search a number of electronic databases including PubMed, MEDLINE, Web of Science, EMBASE, the Cochrane Library (Cochrane Database of Systematic Reviews) and the Cochrane Central Register of Controlled Trials (CENTRAL). We will carry out a secondary search for articles from references of included articles (from January 1990 to June 2014). Primary and secondary outcomes will be BM and skeletal-related events information. We will summarise the effect estimates of risk factors and use random-effect models to pool the estimates, if the outcomes and characteristics in studies are comparable. The quality of the study will be assessed using the Newcastle-Ottawa Scale and the Cochrane Collaboration tool.

Trial registration number: CRD42013003744.

\section{INTRODUCTION}

Bone is a common site of metastasis in lung cancer patients. ${ }^{1}$ Approximately $30-40 \%$ of patients with advanced lung cancer will have bone metastases during the course of their disease, leading to a substantially negative effect on both morbidity and survival. ${ }^{2}$ Bone metastasis $(\mathrm{BM})$ is attributable to the rich blood flow to the bone marrow, high expression levels of adhesional molecules on the malignant cells, and a number of growth factors in the osseous tissue. ${ }^{1} \mathrm{BM}$ in patients with primary lung cancer will lead to skeletal complications, referred to as skeletal-related events (SREs), including pathological fractures, spinal cord compression, hypercalcaemia and severe skeletal pain. Each of these complications may considerably reduce quality of life. ${ }^{34}$

It is important to identify risk factors of BM in patients with primary lung cancer. BM is underdiagnosed globally; a large number of patients have at least one SREs in their lifetime. The increasing number of lung cancer survivors suggests that suffering from SREs might be prevalent. $^{5}$ It is very expensive to treat SREs, and early treatment would be useful to reduce skeletal complications. ${ }^{5}$ Therapies such as bisphosphonates and denosumab might improve quality of life and save money. BM accounts for 350000 cancer patients deaths each year. ${ }^{6}$ Lung cancer was the first cause of death among Chinese cancer patients in 2008. In the past several decades, the mortality rate of lung cancer has risen rapidly; it poses a great threat to human health. ${ }^{7}$

Many advanced measures for early detection of BM are being developed, but it is still hard to identify patients who have an elevated risk of $\mathrm{BM}$. Researchers need comprehensive information to establish a prediction model which will help clinicians provide patients with early treatments. ${ }^{8}$

There have been some studies of risk factors of $\mathrm{BM}$ in patients who have primary lung cancer. However, these studies all focus on considerably narrow fields. We cannot simply synthesise their results, because patients have other systemic diseases and different characteristics. ${ }^{9} 10$ Previous studies have shown that expression of some biochemical compounds (ie, bone sialoprotein, osteopontin and N-telopeptide of type I collagen (NTX), and serum cross-linked carboxyterminal telopeptide of type I collagen (ICTP) and the aminoterminal propeptide of type I collagen (PINP)) are strongly associated with development and progression of $\mathrm{BM}$ in lung cancer patients. ${ }^{8} 11-16$ In order to determine whether these risk factors are relevant, we intend to summarise them and establish a prediction model of BM. We will therefore carry out a systematic review and meta-analysis to determine the potential risk factors and analyse the pooled effects estimates. 


\section{METHODS}

Our review team includes search experts, clinical researchers and systematic review experts.

We develop the methods for this review according to recommendations from the Preferred Reporting Items for Systematic Reviews and Meta-Analyses (PRISMA) ${ }^{17}$ and the Meta-analysis Of Observational Studies in Epidemiology (MOOSE) group. ${ }^{18}$

\section{Criteria for considering studies for this review Study design}

Only case-control, prospective and retrospective cohort studies, randomised controlled trials (RCTs) and systematic reviews will be considered. We will exclude studies without a control group. We will exclude animal experimental studies, simulation studies, studies of screening tests, attitudes, case reports, case series, cross-sectional studies, controlled clinical trials and reviews.

\section{Participants}

Inclusion: primary pulmonary malignancies of adults and elderly people (over 70 years of age), such as non-smallcell lung carcinoma ${ }^{19}$ and small-cell lung carcinoma, ${ }^{20}$ diagnosed using any recognised diagnostic criteria. ${ }^{19} 20$ The diagnostic criteria are recorded in the article.

Exclusion: Adolescents (under 18 years of age); uncertain or unknown information of population; studies only on animals; primary malignancies are uncertain; unclear/unknown diagnostic criteria.

\section{Exposures}

Studies will be considered if they provide adequate description of the risk factors. Potential risk factors will involve clinical features, histological type, biomarkers and genetic characteristics. Clinical features will involve population features (ethnicity, sex, age and BMI), lifestyle (alcohol use or smoking), history of other diseases, Karnofsky score, PS (performance status; Zubrod-ECOG$\mathrm{WHO}$ ), ${ }^{21}$ pain score (such as NRS), systemic disorders (such as diabetes, hypertension or cardiovascular disorders) and treatment.

Articles that can calculate or provide odd ratios (ORs)/relative risks (RRs)/HRs of $\mathrm{BM} / \mathrm{SREs}$ will be considered.

Exclusion: inadequate description of the potential risk factors; unclear results; neither provide nor calculate ORs/ RRs/HRs. Conflicts will be resolved through consensus.

\section{Comparators}

For case-control studies, the comparators of interest are participants with the same diagnosis of primary lung cancer, but without BM/SREs. For cohort studies and RCTs, the comparators of interest are participants with the same diagnosis of primary lung cancer, but without BM/SREs.
Study outcomes

\section{Primary outcome}

The primary outcome will be BM. It will be defined as one or more radiographically confirmed bone metastases. Diagnostic methods include plain radiography, myelography, MRI, CT, radionuclide bone scanning (scintigraphy with technetium-99m-labelled diphosphonates), single-photon emission CT and positron emission tomography. ${ }^{22}$

\section{Secondary outcomes}

The secondary outcomes of this review will be SREs ${ }^{23}$ (including pathological fracture, spinal cord compression, the need for palliative radiotherapy or surgery to bone, and hypercalcaemia of malignancy). ${ }^{4} 23$ This involves appearance of the first SRE, new SREs, skeletal progression and death, with exposures compared with comparators. The articles need to include specific information on performance of the diagnostic test during the studies, including testing at baseline and repeated testing, blinding, and definition of disease progress.

\section{Search strategy for the review \\ Electronic searches}

We will search a number of electronic databases including PubMed, MEDLINE, Web of Science, EMBASE, the Cochrane Library (Cochrane Database of Systematic Reviews) and the Cochrane Central Register of Controlled Trials (CENTRAL), and we will run a secondary search to obtain articles from the references of included articles (from January 1990 to June 2014).

\section{Search strategy}

Experienced information experts will conduct the literature search. We will use three themes to run a search: 'primary pulmonary neoplasm', 'risk factors' and 'bone metastasis'.

The search strategy for PubMed is described in detail in online supplementary file 1 . We will apply a few minor adjustments of the search terms in other bibliographic databases in combination with specific filters, where they are available. No language of publication limitation will be applied. If needed, we will contact a translator.

\section{Selection of studies}

There are several steps to select eligible studies.

1. List the articles which have been identified by the search in Endnote (Microsoft, Redmond, Washington, USA). We will use its function 'remove duplicates' to exclude duplicates.

2. Two review authors (W-WS, Y-TW) will separately examine titles and abstracts to exclude the obviously irrelevant reports. In case of doubt, proceed to the next step.

3. Get the full text of papers in the first list through the internet and authors of reports.

4. Put articles of the same research together. 
5. Two authors (W-WS, Y-TW) will separately screen fulltext articles according to eligibility criteria.

6. If required, we will contact the authors of the reports for further information to clarify study eligibility.

7. Make a final list and proceed to the next stage. We will present our selection process in a flow chart, keep an account of studies excluded and record justifications in the review.

We will exclude abstracts published in conference proceedings. As blinded selection may be impossible, we will conduct an unblinded selection. Conflicts will be resolved by discussion among three authors, including a methodologist.

\section{Data collection}

Two reviewers (W-WS, Y-TW) will separately present data extraction using a unified collection form. After June 2014 we will complete the search for studies, and start to check these studies. At the end of selection, our team will start to collect data. Collecting information may be hampered by incomplete reporting of the studies. If needed, we will contact authors using an open-ended questionnaire which will reduce the risk of overly positive answers. Conflicts will be resolved by discussion among three authors, including a methodologist.

The following information will be collected:

1. Identification: study ID; article ID; review author ID.

2. Eligibility: confirmation; exclusion reason.

3. Methods: design; study period; inclusion/exclusion criteria; comparators setting.

4. Participants: setting of population; mean age; race; gender distribution.

5. Exposures (risk factors): number of exposure groups.

For each exposure and comparator group:

Specific definition; measurements; potential confounding factors.

6. Outcomes: outcomes and time points collected; reported.

For each outcome:

Definition; total number of participants; number experiencing the outcome in each group; number analysed; number of BM/SREs; BM (primary site of BM; number of bone metastases; histological type of primary lung cancer; definition of the BM; diagnostic test of bone metastases); SREs (diagnosis tests of SREs; appearance time of first SRE; new SREs; skeletal progression; deaths).

7. Results: number of participants in each group.

For each outcome:

Sample size; missing participants; adjusted/unadjusted effect estimates (RRs/ORs/HRs) and their SEs/CIs; subgroup analyses; covariates (if estimates adjusted for covariates (such as systematic disorders and PS), write them down).

8. Methodology: exclusion rate; whether addressed or unaddressed sampling bias; methods used to synthesise data; methods used to analysis data.

9. Conclusion: key conclusion; comments by reviewers.
10. Publication: title; first author; year and language of publication.

\section{Assessment of risk of bias}

We will use the Newcastle-Ottawa scale ${ }^{24}$ to assess the risk of bias of observational designs, and the Cochrane Collaboration tool $^{25}$ will be adopted to value the risk of bias of RCTs. Conflicts will be resolved through consensus among three authors, including a methodologist. If the missing data leading to the study quality cannot be synthesised or assessed, we will contact the authors using an open-ended questionnaire for complete information. We will exclude the study if there is still unclear or unknown information after this step.

\section{Statistical analysis}

Data synthesis and analysis

We will summarise the individual studies as well as the results of systematic reviews, and present the factors that may change BM risk in figures. If some studies have the same risk factor, similar comparators and consistent outcome measure (BM/SREs), we will perform a random-effect meta-analysis using the HRs/ORs/RRs to estimate the pooled effect estimates and 95\% CIs. Considering the substantial difference, which is made by switching the effect estimates (HRs/ORs/RRs), these estimates will be analysed independently. When the data are adequate, we will adjust the overall estimates for potential confounding factors. Vote counting will be regarded as a last resort, when standard meta-analysis cannot be applied. To assess the quality of evidence in studies, we will adopt the principles of the GRADE system. $^{26}$

\section{Assessment of heterogeneity}

We will use the I-squared statistic to quantify inconsistency across studies. If the observed value is greater than $50 \%$, we will consider that it may represent substantial heterogeneity. We will incorporate heterogeneity into random-effects meta-analysis to estimate the variance of exposure effect across studies (ie, the $\tau^{2}$ statistic). To search for interactions (such as populations and exposure features), we will undertake subgroup analyses for exploring study characteristics (ie, study region). Moreover, sensitivity analyses will be performed for identifying unclear decisions that greatly influence the results. If these unclear decisions cannot be resolved, we will give a cautious explanation of the results.

\section{Reporting biases}

In addition to two approaches of potentially reducing reporting biases (ie, comprehensive search strategy and trial registry), we will present funnel plots for a visual impression of publication bias.

All statistical analyses will be performed with Stata V.11 and Review Manager (Revman V.5.2). All statistical tests will be two-sided. 


\section{DISCUSSION AND DISSEIMINATION}

Our findings from this systematic review will help us generate a basic conceptual structure to better understand the relationship between potential risk factors and BM. Also, we anticipate that these findings will provide comprehensive evidence to help clinicians carry out individualised treatments, and provide researchers with data for establishing a prediction model of BM in patients with primary lung cancer.

This review may have some limitations. Our study may be easily influenced by threats to credibility (ie, internal validity) and applicability (ie, external validity). To appraise the internal validity of studies appropriately, we will assess the bias as much as possible to reduce the risk. For applicability, we will be extremely cautious when making a general statement from one context to another.

The dissemination of this review will involve the sharing of papers among colleagues, presentations at conferences and publishing papers in journals.

Acknowledgements The authors wish to record their appreciation to Wen-Min Niu, Doctor of Medicine at the College of Shaanxi Chinese Medicine for his advice and to Dong-Lei Jiang, Professor of The Fourth Military University, for helping us revise this manuscript.

Contributors Y-JN and H-LZ are responsible for the generation of the research question and design of the protocol, and will lead and oversee the study. Y-JN drafted the manuscript. LD and L-LL assisted with the design of protocol, and Y-TW provided guidance for developing the search strategy. W-WS helped draft the manuscript and provided comments. All authors read and approved the final manuscript.

Funding This study was supported by National Natural Science Foundation of China (no. 81172011; 81272348).

Completing interests None.

Provenance and peer review Not commissioned; externally peer reviewed.

Data sharing statement The systematic review protocol is registered with the PROSPERO International Prospective Register of Systematic Review (http:// www.crd.york.ac.uk/prospero) (registration number: CRD42013003744) and reported using the Preferred Reporting Items for Systematic Reviews and Meta-analysis (PRISMA) guidelines in the peer-reviewed literature. The systematic review will be in one manuscript and shared with others.

Open Access This is an Open Access article distributed in accordance with the Creative Commons Attribution Non Commercial (CC BY-NC 4.0) license, which permits others to distribute, remix, adapt, build upon this work noncommercially, and license their derivative works on different terms, provided the original work is properly cited and the use is non-commercial. See: http:// creativecommons.org/licenses/by-nc/4.0/

\section{REFERENCES}

1. Roodman GD. Mechanisms of bone metastasis. $N$ Engl J Med 2004;350:1655-64.

2. Al Husaini HMD, Wheatley-Price PMM, Clemons MMF, et al. Prevention and management of bone metastases in lung cancer: a review. J Thorac Oncol 2009;4:251-59.
3. Bae HM, Lee SH, Kim TM, et al. Prognostic factors for non-small cell lung cancer with bone metastasis at the time of diagnosis. Lung Cancer 2012;77:572-7.

4. Coleman RE. Clinical features of metastatic bone disease and risk of skeletal morbidity. Clin Cancer Res 2006;12(20 Pt 2):6243s-49s.

5. Langer C, Hirsh V. Skeletal morbidity in lung cancer patients with bone metastases: demonstrating the need for early diagnosis and treatment with bisphosphonates. Lung Cancer 2010;67:4-11.

6. Mundy GR. Metastasis to bone: causes, consequences and therapeutic opportunities. Nat Rev Cancer 2002;2:584-93.

7. She J, Yang $P$, Hong $Q$, et al. Lung cancer in China: challenges and interventions. Chest 2013;143:1117-26.

8. Zhang L, Hou X, Lu S, et al. Predictive significance of bone sialoprotein and osteopontin for bone metastases in resected Chinese non-small-cell lung cancer patients: a large cohort retrospective study. Lung Cancer 2010;67:114-19.

9. Coleman RE. Skeletal complications of malignancy. Cancer 1997;80 (8 Suppl):1588-94.

10. Sekine I, Nokihara $\mathrm{H}$, Yamamoto $\mathrm{N}$, et al. Risk factors for skeletal-related events in patients with non-small cell lung cancer treated by chemotherapy. Lung Cancer 2009;65:219-22.

11. Papotti $M$, Kalebic $T$, Volante $M$, et al. Bone sialoprotein is predictive of bone metastases in resectable non-small-cell lung cancer: a retrospective case-control study. J Clin Oncol 2006;24:4818-24.

12. Huang $Q$, Ouyang $X$. Biochemical-markers for the diagnosis of bone metastasis: a clinical review. Cancer Epidemiol 2012;36:94-8.

13. Kobayashi T, Gabazza EC, Taguchi O, et al. Type I collagen metabolites as tumor markers in patients with lung carcinoma. Cancer 1999;85:1951-7.

14. He JJ, Zhi K, Liu GF. Predictive value of serum bone sialoprotein in patients with bone metastasis of non-small cell lung cancer. Onkologie 2011;34:584-8.

15. Brown JE, Cook RJ, Major $P$, et al. Bone turnover markers as predictors of skeletal complications in prostate cancer, lung cancer, and other solid tumors. J Natl Cancer Inst 2005;97:59-69.

16. Coleman RE, Major $\mathrm{P}$, Lipton $\mathrm{A}$, et al. Predictive value of bone resorption and formation markers in cancer patients with bone metastases receiving the bisphosphonate zoledronic acid. J Clin Oncol 2005;23:4925-35.

17. Moher D, Liberati A, Tetzlaff J, et al. Preferred reporting items for systematic reviews and meta-analyses: the PRISMA statement. PLoS Med 2009;6:e1000097.

18. Stroup DF, Berlin JA, Morton SC, et al. Meta-analysis of observational studies in epidemiology: a proposal for reporting. Meta-analysis Of Observational Studies in Epidemiology (MOOSE) group. JAMA 2000;283:2008-12.

19. Goldstraw $P$, Ball D, Jett JR, et al. Non-small-cell lung cancer. Lancet 2011;378:1727-40.

20. van Meerbeeck JP, Fennell DA, De Ruysscher DK. Small-cell lung cancer. Lancet 2011;378:1741-55.

21. de Kock I, Mirhosseini M, Lau F, et al. Conversion of Karnofsky Performance Status (KPS) and Eastern Cooperative Oncology Group Performance Status (ECOG) to Palliative Performance Scale (PPS), and the interchangeability of PPS and KPS in prognostic tools. J Palliat Care 2013;29:163-9.

22. Sutcliffe $P$, Connock $M$, Shyangdan $D$, et al. A systematic review of evidence on malignant spinal metastases: natural history and technologies for identifying patients at high risk of vertebral fracture and spinal cord compression. Health Technol Assess 2013;17:1-274.

23. Lipton A, Cook R, Brown J, et al. Skeletal-related events and clinical outcomes in patients with bone metastases and normal levels of osteolysis: exploratory analyses. Clin Oncol ( $R$ Coll Radiol) 2013;25:217-26.

24. Stang A. Critical evaluation of the Newcastle-Ottawa scale for the assessment of the quality of nonrandomized studies in meta-analyses. Eur J Epidemiol 2010;25:603-05.

25. Higgins JP, Altman DG, Gotzsche PC, et al. The Cochrane Collaboration's tool for assessing risk of bias in randomised trials. BMJ 2011;343:d5928.

26. Guyatt $\mathrm{GH}$, Oxman AD, Vist GE, et al. GRADE: an emerging consensus on rating quality of evidence and strength of recommendations. BMJ 2008;336:924-6. 